\title{
Simultaneous estimation of boundary conditions and material model parameters
}

\author{
Gerhardus J. Jansen van Rensburg Schalk Kok Daniel N. Wilke
}

\begin{abstract}
Room temperature experimental compression test data is available for different hardmetals. This data indicates the presence of some spatial inhomogeneity due to a compression instability, eccentric loading or time varying equivalent bending moment. To account for this, an inverse analysis is employed that determines not only the constitutive material model parameter values but also the displacement boundary conditions that best replicate the experimental data. The unknown boundary displacement history is approached using a systematically refined piecewise linear approximation, determined alongside material parameter values. The systematic simultaneous estimation of material parameter values and boundary approximations is also investigated using a virtual problem for which the exact solution is known. This investigation confirms that known material parameter values and boundary conditions can be recovered without using any prior knowledge of the exact displacement boundary conditions.
\end{abstract}

\section{Introduction}

Ideally, experimental material testing is done by subjecting a material sample of basic geometry to a simple, repeatable and well behaved load case. The parameter values of a constitutive description need to be chosen in such a way that the model best replicates the experimentally observed response when simulating the experiment. If the experiment can be modeled using a closed form analytical expression, the setup of the material parameter characterization process is simple.

Gerhardus J. Jansen van Rensburg

(1) Modelling and Digital Science, Council for Scientific and Industrial Research, Pretoria, South Africa

(2) Computer Science and Applied Mathematics, University of the Witwatersrand, Johannesburg, South Africa

Contact e-mail: jjvrensburg@csir.co.za

Schalk Kok and Daniel N. Wilke

(3) Centre for Asset Integrity Management, Department of Mechanical and Aeronautical Engineering, University of Pretoria, Pretoria, South Africa

Contact e-mail: nico.wilke@up.ac.za·schalk.kok@up.ac.za
In the case for soft metals the ideal compressive test comprises a cylindrical test specimen compressed between two harder dies or plates. The specimen contact surfaces could be grooved and lubricated to prevent rollover and subsequent inhomogeneous deformation. The goal is to obtain as uniform a stress state as possible so that the material characterization may be done using simple post-processing on an easily obtained stress - strain curve. Various standards and procedures are in place for these tests under various conditions (ASTM E209-00, 2010; ASTM E9-09, 1989). When using experimental data with a uniform distribution in stresses, constitutive model parameters may then be determined following an inverse problem approach. Bruhns and Anding (1999) effectively illustrated this using a least squares criterion for different model formulations and optimization methods. This is also useful for complex material models where a uniform distribution in stresses may be assumed as an initial or first order estimate of material properties (Jansen van Rensburg, 2016; Jansen van Rensburg et al., 2017).

The monolithic compression testing of hard material samples at room temperature also has its different standards and procedures (ASTM C1424-04, 2004; Dunlay et al., 1989; ISO 4506, 1979). An unfortunate reality of hardmetal testing is due to the high stiffness of the specimen compared to the effective stiffness of the test machine. This may result in a material test that does not conform to the ideal loading condition. The resulting non-uniform stress state reduces the efficacy of closed form post-processing. In this paper, experimental data (load cell force data and strain gauge readings) for a number of compression tests are available at room temperature for different grades of cemented Tungsten Carbide (WC-Co) or hardmetal samples. The different material grades have varying particulate sizes, binder phase chemistry and percentage volume fractions. The available experimental compression data indicates that the samples were subjected to non-ideal compression that resulted in inhomogeneous deformation. The exact displacement conditions that resulted in the inhomogeneity are however unknown.

In cases where the experimental material testing were not ideal, a more detailed inverse modeling approach to closed form post-processing needs to be considered. The parameter identification of inelastic material models based on experimental data for specimens with nonuniform stresses and strains (Mahnken and Stein, 1996) are of particular relevance to this paper. Within this 
approach an objective function is constructed as some measure of agreement between the measured experimental data and Finite Element Analysis (FEA) simulated equivalent data.

Ghouati and Gelin (1998) characterize their material model directly from simulations on a deep drawing operation of interest. The simulation parameters are adjusted within an inverse analysis so that the calculated response best match their measured punch load and displacement of the punch during loading. Wikman et al. (2006) on the other hand characterized the constitutive parameter values of a rate-independent elastoplastic associative cap material model used to simulate a compacting powder. They performed an inverse analysis on a compacting experiment using a modified version of the downhill simplex method.

Åkerström et al. (2005) characterize Boron steel in compression. An axisymmetric simulation of their Gleeble compression experiment is done subjected to variations of the 8 unknown parameter values in their rate independent thermo-elastoplastic model. They performed an inverse analysis using anvil displacement taken during their three different simultaneous cooling and compression experiments at different rates as known and applied it as appropriate boundary conditions. They constructed an objective function that compares the Finite Element solution and sample points of experimental data in a least squares sense. Their unconstrained inverse problem is solved using a direct search method. Wang et al. (2017) included the effect of barreling during Gleeble compression on their effective stress-strain curve for low carbon steels. Their inverse problem encompasses a range of temperatures and strain rates. They account for the affect of Coulomb friction by considering different friction coefficients to obtain the actual stress-strain curves needed to replicate their experimental observations.

Schmaltz and Willner (2014) investigate the usability of different biaxial test specimen geometries and loading conditions to identify the plastic material parameters of sheet metal. They solve an inverse problem by simulating different biaxial tests performed on sheet metal to characterize an elastoplastic material model with Hill-type yield. Their objective function is again constructed to fit experimental response in a least squares sense. They investigate the use of three geometrical designs for biaxial test specimens under varying levels of heterogeneity in the resulting strain and stress fields. They had access to full field deformation measurements as determined from a two camera system with displacements calculated via digital image correlation in addition to the measured forces using four load cells. Starting with the same three initial parameter value sets they solved the inverse problems to see if all three runs reach the same optimal parameter set or global optimum. The four forces measured were considered to be known as well as the applied boundary conditions while they compare the calculated dis- placements to the measured full field displacements in a least squares sense. From their study, Schmaltz and Willner (2014) showed that the identification procedure fails when more effects are present than their material model can simulate. If all effects can be modeled however, it is more beneficial to have experiments with more heterogeneous deformation. Simultaneous geometric effects, tension, compression and shear stresses were shown to enrich the data set for finite element based inverse identification in their case.

Material hardness and indentation tests are other examples illustrating the application of FEA based inverse modeling to elastoplastic material parameter characterization. Gamonpilas and Busso (2007) validated their proposed elastoplastic characterization using a virtual indentation experiment. They performed the inverse characterization using pseudo-experimental load-displacement data for two indenter designs. They showed that using only the load-displacement data of a single indenter could result in an ill-conditioned problem. This issue was resolved when they used both data sets simultaneously. Chen et al. (2017) also illustrated the use of FEA based inverse analysis to characterize the elastoplastic response of aluminum over different length scales using indentations of varying size.

Ageno et al. (2009) and Garbowski et al. (2012) performed inverse FEA modeling of foil experiments to determine the material parameters for chosen material models. Specifically, Garbowski et al. (2012) considered orthotropic elastic-plastic constitutive models characterized by inverse modeling of biaxial cruciform tests. On the other hand, Ageno et al. (2009) characterized the elastic-plastic response of bulging thin membranes subjected to an experimentally controlled fluid pressure. Similarly, Jekel et al. (2016) performed inverse analyses of their proposed bubble inflation test for non-linear orthotropic membrane materials. The effective FEA based inverse characterization of their experimental setup was illustrated using a virtual problem or pseudo-experimental data.

In this paper, a detailed FEA based inverse characterization on the hardmetal compression experiments is performed. With the experimental displacement boundary conditions unknown, the material parameter values and displacement history are estimated simultaneously. In Section 2 the experimental data and material model are discussed. By assuming that the effects of inhomogeneous deformation are negligible, simple post-processed inverse material characterization is discussed in Section 3. The simple post-processed material parameter values are used for comparison later on. This is followed by a detailed FEA based inverse characterization on one of the data sets in Section 4. The finite element method is used to simulate the non-ideal compression tests subject to an unknown displacement boundary condition, modeled using a piecewise linear approximation. To test the validity and efficacy of this approach, the procedure is also performed on a set of 


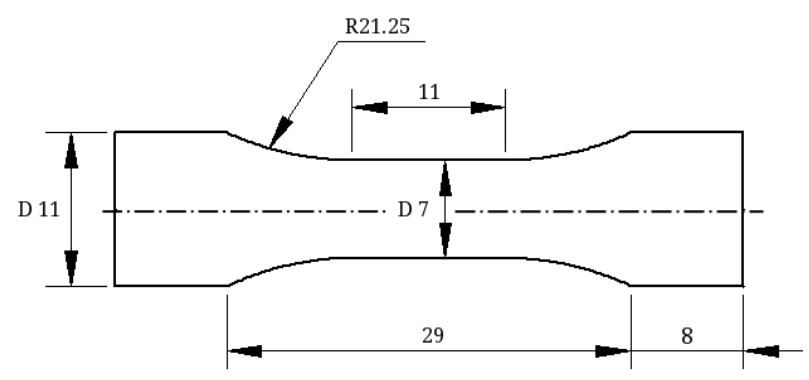

Figure 1: Dimensions of the $45 \mathrm{~mm}$ modeled cylindrical test specimen.

virtual or pseudo-experimental data in Section 5 modeled on the test case presented in Section 4. Section 6 treats the remainder of the experimental data and conclusions are provided in Section 7 .

\section{Experimental Data and Mate- rial Model}

\subsection{Experimental Data}

The data and work in this chapter is subject to an ongoing memorandum of understanding where the data is proprietary information and as such may not be published as is. The alloy compositions and chemistry are therefore not reported and in figures where the data is presented, the values of the ordinate axis is often removed or scaled to comply with the memorandum of understanding. The experimental room temperature compression test data for this paper is obtained from 3 strain gauges around the cylindrical test specimen, along with hydraulic cylinder displacement and load cell data at regular time intervals. The strain gauges are fixed to the center of the cylindrical test specimen, $120^{\circ}$ apart.

A material test specimen similar to the one described by Dunlay et al. (1989) is used for the experimental compression tests. The compression test specimen is essentially a modified version of a conventional cylindrical tensile test specimen. The length of the test section is reduced in an attempt to avoid buckling and shear deformation modes during the compression test. Figure 1 depicts the dimensions of the $45 \mathrm{~mm}$ cylindrical test specimen.

One of the demanding aspects of compression tests on these hard materials is the very high compressive strength and stiffness of the specimens. The testing machine stiffness, which should ideally be orders of magnitude greater than that of the specimen, is inadequate in this case. Experimental data indicates that for a typical test the hydraulic cylinder displaces $1.4 \mathrm{~mm}$, while the test specimen only decreases in height by about $0.4 \mathrm{~mm}$. Consequently, elastic deformation of

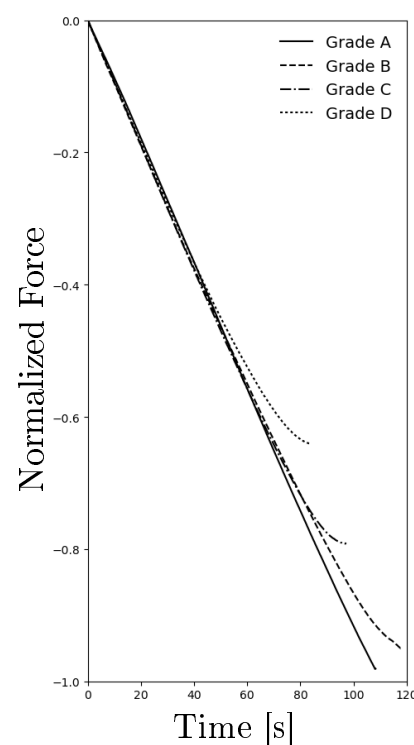

(a)

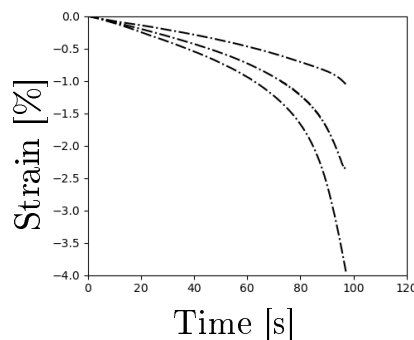

(d)

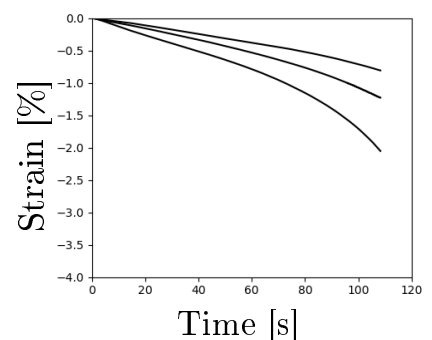

(b)

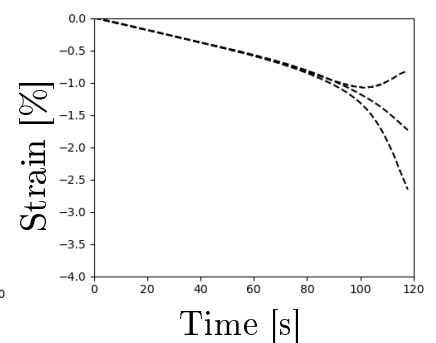

(c)

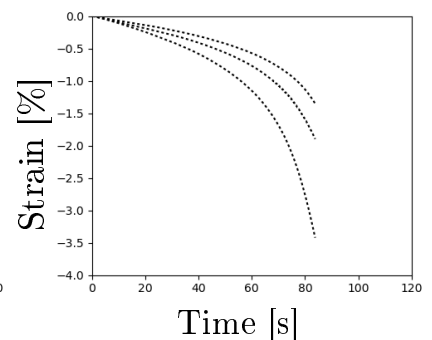

(e)
Figure 2: Normalized force and strain data. (a) The force history of four different hardmetal grades named Grade A to D. The strain histories extracted at three locations $120^{\circ}$ apart around the test specimen center for Grades (b) A, (c) B, (d) C and (e) D show the presence of an eccentric load, compressive instability or time varying equivalent bending moment.

the testing machine frame accounts for the remaining $1.0 \mathrm{~mm}$ displacement.

The experimental compression test data histories for four different hardmetal grades and composition are given in Figure 2. Because of the proprietary nature of the materials the different samples tested are simply distinguished by naming them Grade A to D. Figure 2(a) depicts the normalized force histories for the four grades, while Figures 2(b) to (e) show the three strain gauge histories around each of the four specimens. From the strain gauge data for the different hardmetal grades and composition there appears to be some compressive instability, eccentric load condition or time varying equivalent bending moment present.

The inhomogeneous deformation evident from the strain histories in Figure 2 suggests that a nonuniform stress state develops during the compression test, possibly due to compliance of the test frame. The data also indicates that the strain rate in the specimen is not constant, even though the crosshead speed of the 
hydraulic cylinder is.

\subsection{Material Model}

For numerical modeling purposes, an internal state variable based formulation for the yield stress

$$
\sigma_{\mathrm{Y}}=\sigma_{0}+\hat{\sigma}
$$

is assumed where $\sigma_{0}$ is the yield at zero plastic deformation $\left(\varepsilon_{\mathrm{p}}=0\right)$ and $\hat{\sigma}$ is an internal state variable that represents the evolving yield stress component. The isothermal evolution of $\hat{\sigma}$ follows an empirical modification for the Voce law (Kocks et al., 1998; Mourad et al., 2013) reading

$$
\frac{d \hat{\sigma}}{d \varepsilon_{\mathrm{p}}}=\theta_{0}\left(1-\frac{\hat{\sigma}}{\hat{\sigma}_{S}}\right)^{n} .
$$

Using this material model, there are 4 variables that have to be estimated so that the modeled response best represents the experimental data: an initial yield stress $\left(\sigma_{0}\right)$, initial work hardening rate $\left(\theta_{0}\right)$, saturation stress $\left(\sigma_{S}\right)$ and hardening exponent $(n)$.

The problem statement and solution illustrated in this paper now follows from the task to estimate the material model parameters as accurately as possible. The material model parameters are first estimated from the experimental test data by using simple post-processing. A representative material parameter set and time varying displacement boundary condition are then used to construct a virtual experimental data set using Finite Element Analysis (FEA). Simple post-processing on the virtual experimental data set then gives an indication of the expected estimation error when compared to performing an FEA based estimation.

\section{Simple Post-Processing}

Strain gauge and load cell data for all four hardmetal grades are used here for material parameter estimation using simple post-processing. If simple post-processing of the data is performed, the average values of the strain gauges $\bar{\epsilon}$ at each time step are used to compute the true strain values from

$$
\varepsilon_{\text {true }}=\ln (1+\bar{\epsilon}),
$$

where negative strains indicate compression. Assuming the material is volume preserving, the load cell force value $P$, initial test specimen area $A_{0}$ and engineering strains are used to approximate the true stress value at each time increment using

$$
\sigma_{\text {true }}=\frac{P}{A_{0}}(1+\bar{\epsilon}) .
$$

A plot of the stress strain curves of the four different grades are given in Figure 3(a) along with a straight
Table 1: Material model parameters

\begin{tabular}{lllllll}
\hline & $\begin{array}{l}\text { Average } \\
(\text { Normalized })\end{array}$ & $\mathrm{A}$ & \multicolumn{4}{l}{ (Scaled parameter values $)$} \\
\hline$\sigma_{0}$ & $0.374 \mathrm{MPa}$ & 1.523 & 1.249 & 0.623 & 0.605 \\
$\theta_{0}$ & $0.787 \mathrm{GPa}$ & 1.298 & 1.200 & 0.871 & 0.631 \\
$\sigma_{S}$ & $0.869 \mathrm{MPa}$ & 1.227 & 1.125 & 0.910 & 0.738 \\
$n$ & 1.625 & 1.072 & 1.008 & 0.957 & 0.962 \\
\hline
\end{tabular}

line indicating the normalized elastic modulus of 9.692 MPa. Although the different material grades vary in a number of aspects, the elastic properties seem identical. The expected range of Poisson's ratio is $0.2-0.22$ (2002).

The maximum compressive strength and plastic deformation differ substantially between the material grades. Removing the elastic strain component from the total strain values results in the stress versus plastic strain curves in Figure 3(b). The material model is coded in Python and then calibrated to the simplified test data using the Nelder-Mead simplex algorithm available in SciPy (Jones et al., 2001).

The normalized material parameter values used to model the normalized stress-strain curve for each hardmetal grade in Figure 3(b) is listed in Table 1. This table contains a column for the average material parameter value considering all four grades as well the scaled values per grade to show inter-grade variation.

Following the simple post-processing, an investigation on the material model parameters as a result of detailed FEA based material parameter estimation is now discussed.

\section{Finite element based inverse analysis}

In FEA based material parameter estimation, the experimental test specimen can be modeled subject to a time varying equivalent bending moment and axial displacement. Since the form of the equivalent time varying displacement boundary condition is unknown, it may be approximated using a piecewise linear description as demonstrated on a simple test case in earlier work (Jansen van Rensburg et al., 2012).

In this case, a finite element model is set up by meshing the three dimensional geometry based on the test specimen dimensions in Figure 1. The analysis is performed in CalculiX (Dhondt and Wittig., 1998) using a model with 13056 degrees of freedom and 3696 reduced integration 8 node brick elements. The test spec- 

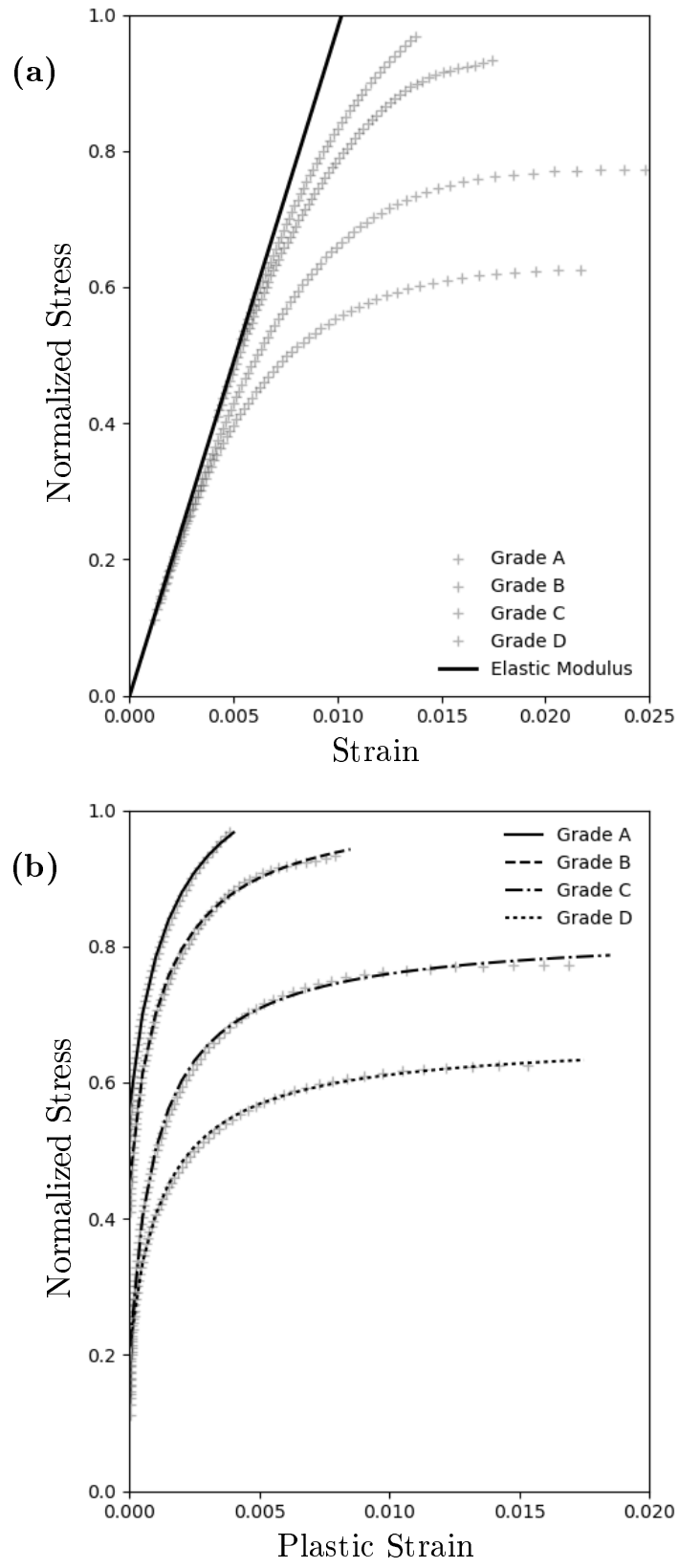

Figure 3: (a) The stress-strain relationship for the various grades of WC-Co using the averaged strains per time increment. The Elastic modulus determined by a linear regression on the stress-strain data below $0.2 \%$ total strain is indicated by the straight black line. (b) The data $(+)$ and modeled (lines) normalized yield stress as a function of plastic strain. imen response is modeled with a material model that has elastic properties as well as plastic behavior using the modified Voce law of Equation (2). This material model is not available within the standard CalculiX material library so it was implemented into a Fortran user material subroutine.

Three elements $120^{\circ}$ or $2 \pi / 3$ radians apart on the circumference of the center of the model are chosen to represent the three strain gauge locations. The strain histories at these locations represent the data associated with the three strain gauges in the actual experiments.

\subsection{Boundary Values}

Time varying axial compression and equal but opposite bending moments at the specimen ends are modeled by prescribing a spatially varied axial displacement field. This is done using a displacement based Multi-Point Constraint (MPC) at the two sides of the modeled test section. The MPC restricts all of the (slave) nodes on either side of the specimen to remain in the same plane subject to the displacement values prescribed at three (master) nodal locations per side. The boundary condition at a given time step is defined by the two boundary properties illustrated in Figures 4(a), (b) and (c) for deviation from the average as well as a time dependent average axial displacement $C(t)$. The displacement field variation $A(t)$ and angle of the neutral axis $B(t)$ can also change as a function of the time. If the axial direction is chosen as the $y$-axis in the finite element analysis, a top face MPC master node $i$ experiences a $y$ displacement of the form

$$
y_{i}(t)=A(t)\left[\sin (B(t)) z_{i}+\cos (B(t)) x_{i}\right]+C(t),
$$

where $x_{i}$ and $z_{i}$ are the $x$ and $z$ coordinates of node $i$. Similarly, the $y$ displacement field at a bottom face node is determined by

$$
y_{i}(t)=-A(t)\left[\sin (B(t)) z_{i}+\cos (B(t)) x_{i}\right] .
$$

By modeling the top and bottom displacement fields in this way, an equal and opposite equivalent bending moment is approximated as a function of time.

The main objective of the FEA based inverse parameter identification remains the estimation of the four material parameters as in Table 1 for the simple postprocessing estimation. In this case however piecewise linear approximations to the boundary functions $A(t)$, $B(t)$ and $C(t)$ are estimated simultaneously.

At the start of the experiment the average axial displacement function $C(t)$ and also deviation from the average $A(t)$ is assumed to be zero, therefore $A^{*}(0)=0$ and $C^{*}(0)=0$. The angle of the neutral axis $B(t)$ on the other hand has a nonzero initial condition. Therefore approximations using $m$ piecewise linear approximations leads to $m$ unknowns for both $A(t)$ and $C(t)$, 


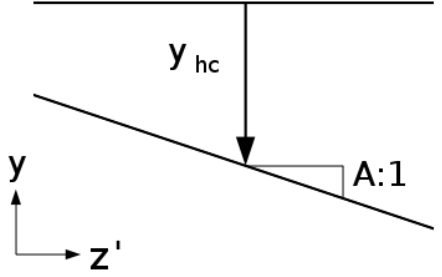

(a)

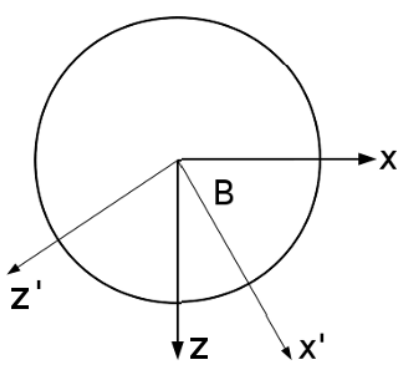

(b)

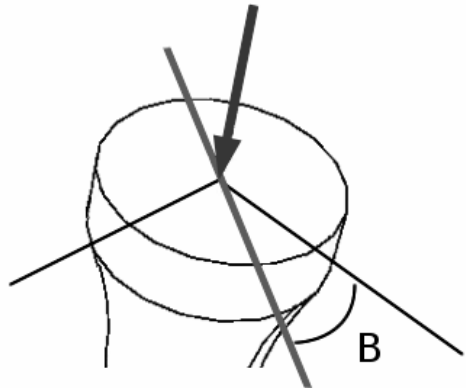

(c)

Figure 4: The two variables $A$ and $B$ that are used to describe the boundary displacement at a specific time. (a) The maximal gradient of the displacement variation is associated with the variable $A$. (b,c) The angle $B$ defines the neutral axis where the effective axial displacement is equal to the average over the entire boundary.

and to $(m+1)$ unknowns in the case for $B(t)$ (to account for the nonzero initial condition). In total, $(3 m+5)$ variables need to be recovered for both the boundary (i.e. $3 m+1$ variables) description and the material model (i.e. 4 variables).

\subsection{Inverse problem characterization and iterative refinement of the piecewise linear boundary approx- imations}

In this section, the simultaneous material parameter and piecewise boundary value function approximations are solved for the strain gauge and load cell data of hardmetal Grade D in Figure 2. The elastic constants are known while the initial yield and plastic hardening parameters are sought as in the simple post-processing case. As initial guess for the unknown material parameters, the values determined from the simple postprocessed data is used. Given that the parameters in Table 1 resulted in the fit to the processed data in Figure $3(\mathrm{~b})$, they are expected to be a reasonable initial guess.

To start, each of the boundary function approximations $A(t), B(t)$ and $B(t)$ are modeled using one unknown. This means that $A(t)$ and $C(t)$ are straight lines starting at zero and ending at unknown final values, while $B(t)$ is an unknown constant. Each of these three unknowns are given an arbitrary initial value. These three initial values are selected manually so that the average and spread of the strains at gauge locations as well as the reaction force are within the range of the experimental data.

Given each guess for the four material parameters and three piecewise boundary function values, an analysis is set up which includes the displacement field boundary conditions of Equations (5) and (6) prescribed over the simulation time at three MPC master nodes per boundary. Once the problem is solved using CalculiX, the objective function used in the inverse analysis optimization procedure quantifies the difference between the finite element simulated curves to the experimental curves. This is done as the sum of absolute fractional errors

$$
f_{\mathrm{obj}}=\sum_{i}\left|\frac{X_{i \text { FEA }}}{X_{i \text { experiment }}}-1\right|,
$$

for each numerically simulated data point $X_{i \text { FEA }}$ of interest compared to the equivalent experimental value

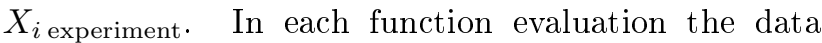
points for the total axial component of the reaction force are compared to the load cell data. The three experimental strain data sets are similarly compared to the associated strain histories extracted from the simulation. The total absolute fractional error thus consists of one force curve error added to three strain curve errors.

All of the data plotted in Figure 2 shows a linear force over time and linear strain over time for the first part of the experiment. From the resulting linear stress vs. strain response also visible in Figure 3, at least the first 10 seconds of the experiment are assumed to be elastic. At least the first 10 seconds of the experiment are assumed to be elastic when considering the force curve in Figure 2(a) and the three strain curves in Figure 2(e) used during this inverse parameter identification. In this case only data points between the 10 second mark and the fully simulated time $(\approx 84$ seconds $)$ are used to calculate the objective function.

As with the simple post-processed parameter estimation, the optimization is performed using the unconstrained optimization algorithm fmin, available via the optimization module in SciPy (Jones et al., 2001). fmin is an implementation of the downhill simplex algorithm (Nelder and Mead, 1965). Although there is access to arguably better numerical optimization algorithms within the SciPy module, fmin is a robust zero order algorithm that only requires function evaluations.

Once the inverse parameter estimation converges, the boundary function approximations are refined. Now two unknowns are assigned to each piecewise linear boundary function approximation before the process is repeated. This means that in the second attempt 
there are still four material parameter unknowns, but now six boundary approximation unknowns, i.e. 10 unknowns in total. The inverse characterization is continued from the previously converged result by placing new unknowns midway between converged piecewise linear point values. Once the ten variable parameter estimation converges, the boundary function approximations are again refined by splitting each piecewise section in two and the process repeats again. The process therefore employs repeated inverse analyses using the same initial setup, where each subsequent stage approximates boundary value functions with higher fidelity.

In this case the simultaneous inverse material parameter and piecewise linear boundary approximation are performed and refined in six stages, ending with an optimization problem where a total of 85 variables are tuned to best fit the data. Of the 85 variables, 81 describe the piecewise linear boundary functions along with the 4 material model parameters.

Figure 5(a) presents the convergence of the error function defined in Equation (7), and Figure 5(b) the number of function evaluations per stage. The initial guess $\left(X_{0}\right)$ results in an objective function value of 1467.66 . The 7 variable inverse characterization requires 460 function evaluations to reach an error of 69.27. The second stage results in an error of 6.942, requiring 1207 additional function evaluations. The trend in a reduced error as a result of additional boundary approximation flexibility in Figure 5(a) continues up to the 25 variable problem with a function value of 0.2443 . Thereafter, for the 45 and 85 variable problems there is little additional improvement in the error. This is despite an additional 2697 function evaluations needed to reach an objective function value of 0.1635 , and a further 2298 function evaluations to reach an error of 0.1581 . Note that each function evaluation requires a single FEA simulation. The total computational cost for the six stage refinement is therefore 11055 independent FEA simulations, shown as the black line in Figure 5(b).

In Figure 6 the strains and total axial reaction force histories for each stage are compared to the Grade D hardmetal experimental data curves. This provides visual representation of the response using the initial conditions $\left(X_{0}\right)$ and convergence towards the experimental data over the various stages of boundary function approximation refinement and parameter identification.

Figures $7(\mathrm{a})$ to $(\mathrm{e})$ depict the convergence of the material model parameters over the different stages. Here again there is visually little change from the 25 variable solution onward. All of the parameter values are scaled using the final value determined using 85 variables as reference. Comparing the initial material parameter values obtained by fitting the model to simple post-processed data, the initial yield stress is finally characterized $16.78 \%$ higher than the value in Table 1. The initial hardening rate is $11.52 \%$ lower, the satura- (a)

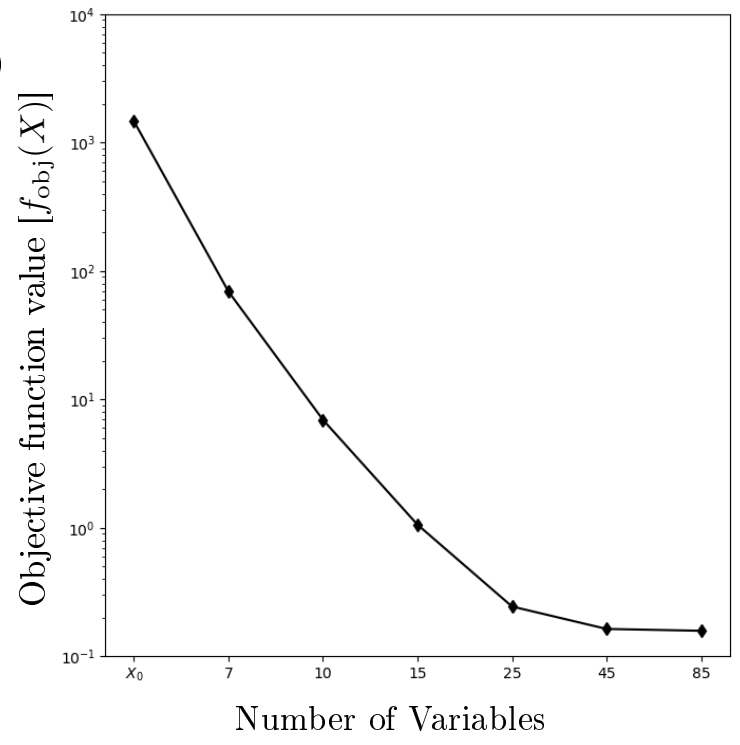

(b)

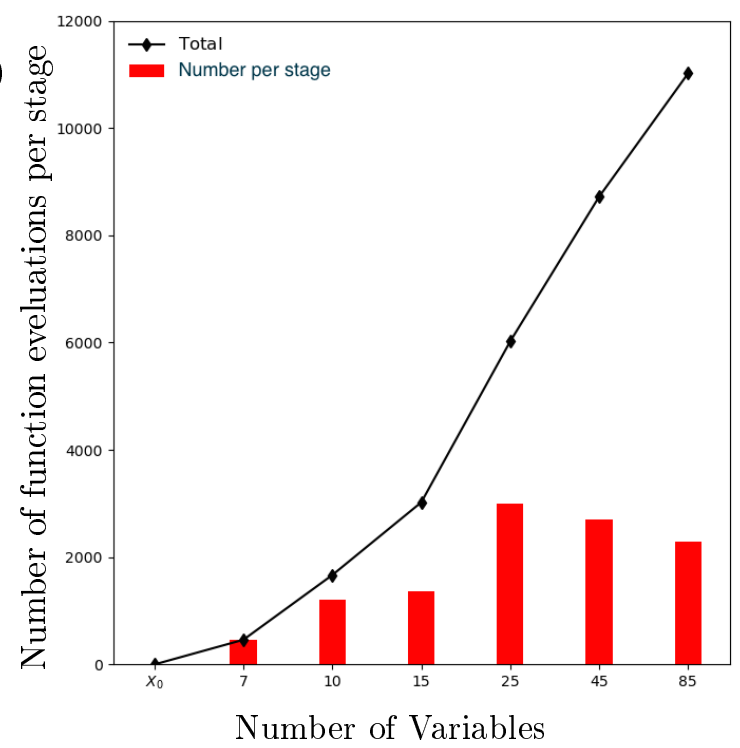

Figure 5: (a) Reduction in best objective function value as the number of variables (i.e. boundary approximation and material parameters) increase for the repeated inverse analyses, and (b) the required number of $\mathrm{FE}$ analyses per run and the cumulative number of FE analyses, to estimate the material parameters and boundary conditions for the Grade D material. 

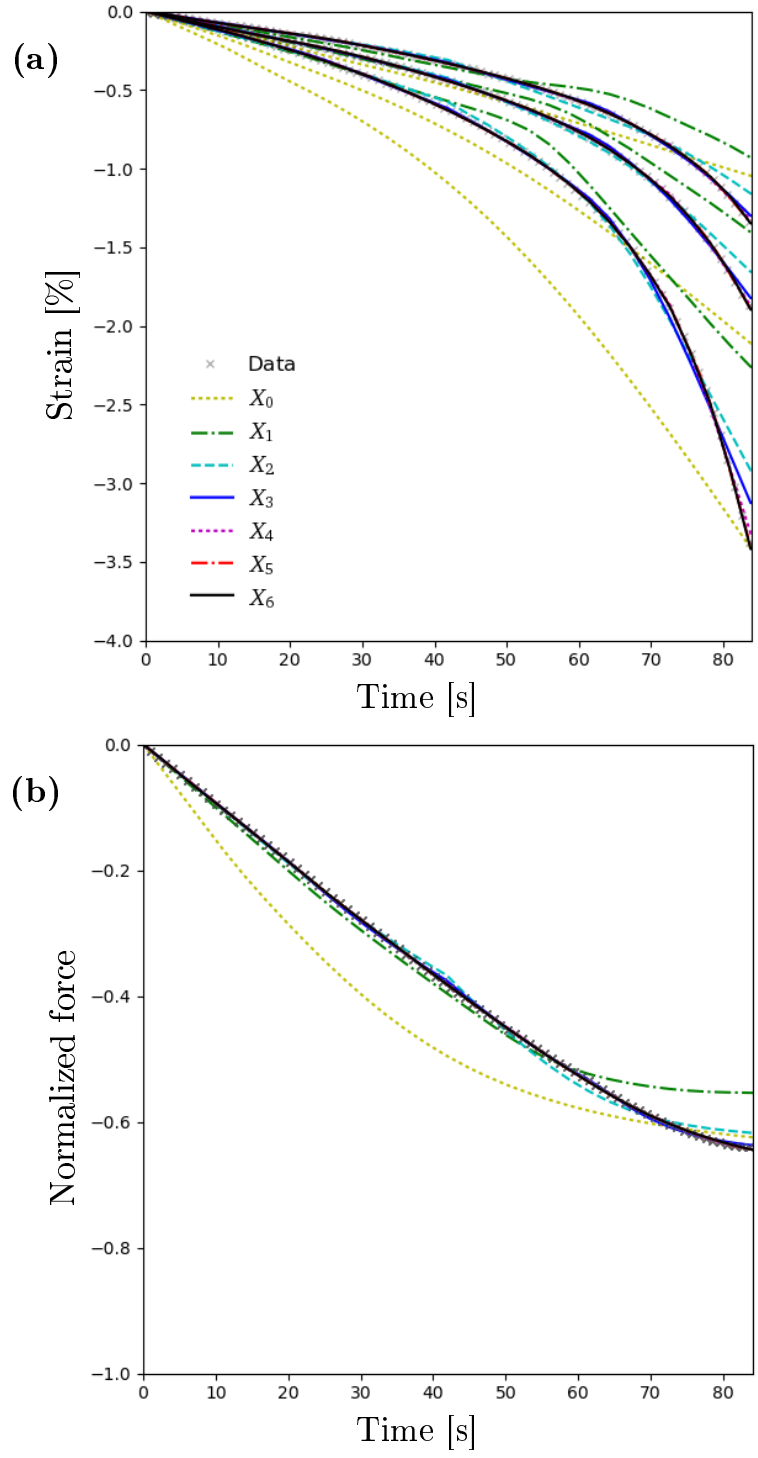

Figure 6: (a) Strains and (b) normalized force histories of the experimental and numerical simulations for the Grade D hardmetal. Better representation of the experimental data is visible as a result of piecewise linear boundary function approximation refinement.

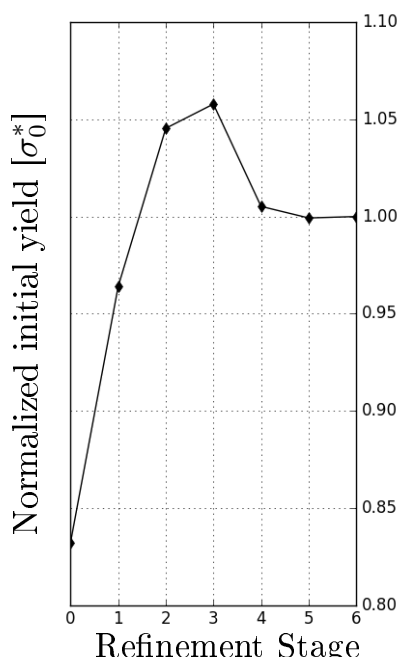

(a)

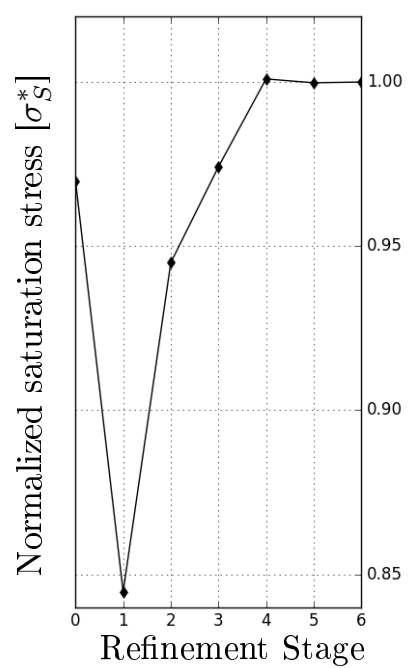

(c)

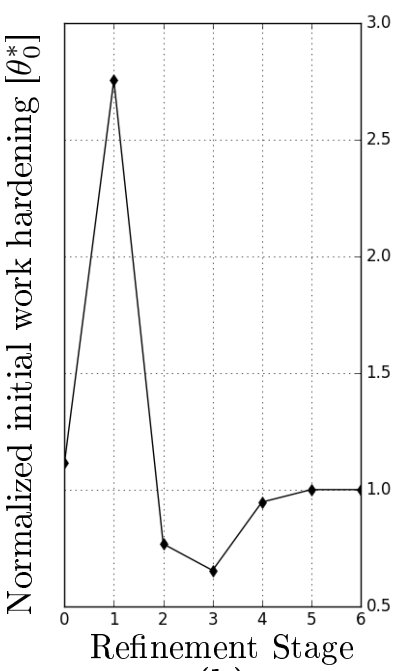

(b)

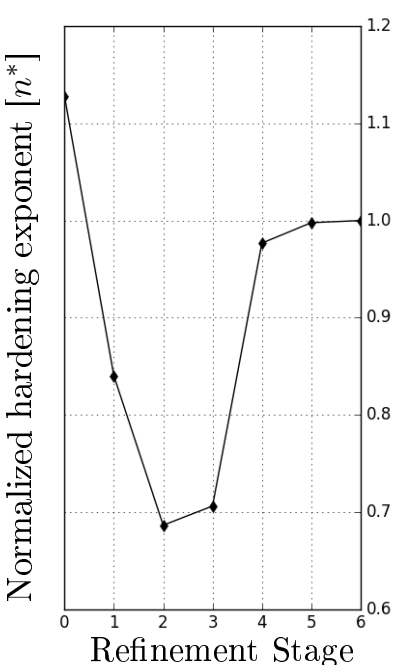

(d)
Figure 7: Material parameter convergence depicted in (a)-(d) as a result of higher modeling flexibility in the piecewise linear boundary value approximations.

tion stress is $3.01 \%$ higher and the hardening exponent is $12.79 \%$ lower compared to the initial guesses respectively.

Considering the 25 variable stage results, the initial yield stress is within $0.52 \%$ of the final 85 variable value. The initial hardening rate is within $5.35 \%$, the saturation stress is within $0.094 \%$ and the hardening exponent within $2.32 \%$ of the final result. In the 45 variable case the largest deviation from the final material parameter value is the initial hardening rate at $2.21 \%$ while all of the other values now vary by less than $0.1 \%$ of the final result. Although not implemented here, this relative change in material parameter estimates from one to stage to another could be used as a convergence criterion.

Figure 8 depicts the form of the boundary function approximations. Here the initial conditions using manually selected straight lines for $A(t)$ and $C(t)$ along with 
the constant for $B(t)$ are seen along with the values and convergence of the initially unknown forms through the various stages of estimation and piecewise linear function refinement.

Figure 9 depicts the contours of equivalent axial strains and normalized Von Mises stresses of the final converged result. From these contours, it is evident that the slightest equivalent bending moment or compressive instability is seen to result in significant variation of stresses and strains in the test section. The process to estimate the final material parameters and detailed piecewise linear boundary value approximations required 11055 FEA simulations. This makes it a computationally expensive exercise.

Another concern of this approach is that a better fit to the experimental data might not necessarily imply a better approximation of the material stress-strain curve. It is conceivable that the complex boundary conditions allow a better fit to the data, but that both the true stress-strain curve and the true boundary condition are approximated with worse accuracy. In essence, the inverse problem could be ill-posed where various combinations of stress-strain curves with its associated boundary condition variation can fit the experimental data well.

It is important therefore to investigate whether the proposed procedure is worth the computational cost. The accuracy with which this approach finds the required material parameter values compared to a simple post-processing characterization is also important. To address these questions, a study is done using virtual experiment data in the following section. Virtual data is extracted from an FEA simulation so that the baseline and true parameter values for comparison are known. The ability of the procedure to find the now known parameter values should give an indication on the accuracy of the procedure.

\section{Investigation using virtual ex- periment data}

The virtual experiment for this study is based on using the forms of the boundary functions observed necessary to replicate the Grade D data in the previous section. Here, the same basic shape as the boundary function forms are parametrized to first investigate whether the fully parametrized virtual problem is invertible or illconditioned based on the data available. Thereafter an iteratively refined piecewise linear approach is followed. This is done to investigate the extent to which the material parameter values and boundary function form can be recovered without information on the exact parameterization.

For the virtual problem, the deviation from the average axial displacement as a function of the time fraction
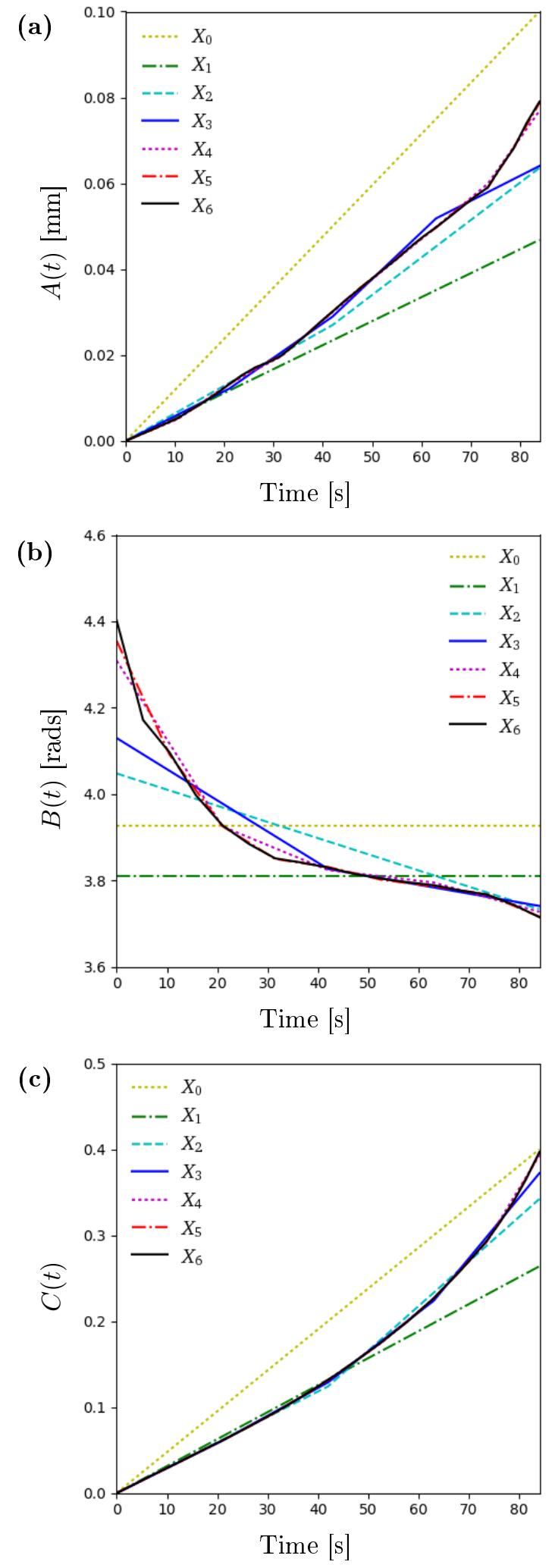

Figure 8: Form of the piecewise linear approximated boundary function approximations. (a) Deviation from the average axial displacement $A(t)$, (b) angle of the neutral bending axis $B(t)$ and (c) axial displacement $C(t)$. 


$$
A(\tau)=A_{0} \frac{\sinh \left(A_{1} \tau\right)}{\sinh \left(A_{1}\right)}
$$

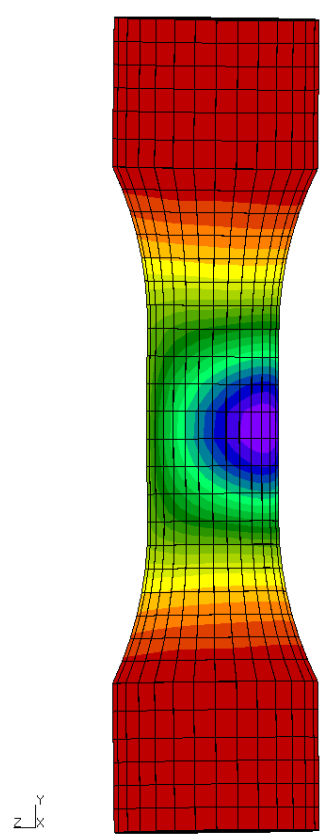

(a)

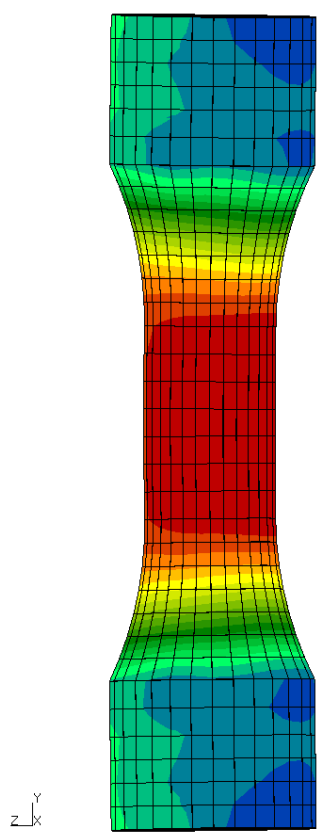

(c)

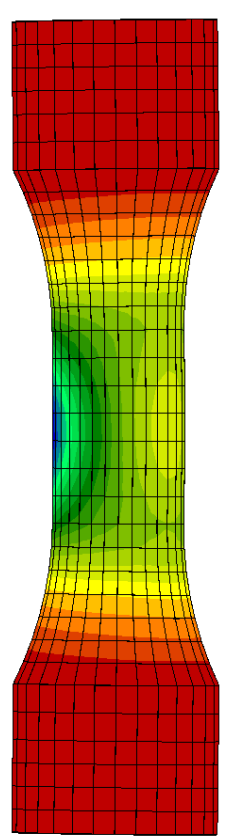

(b)

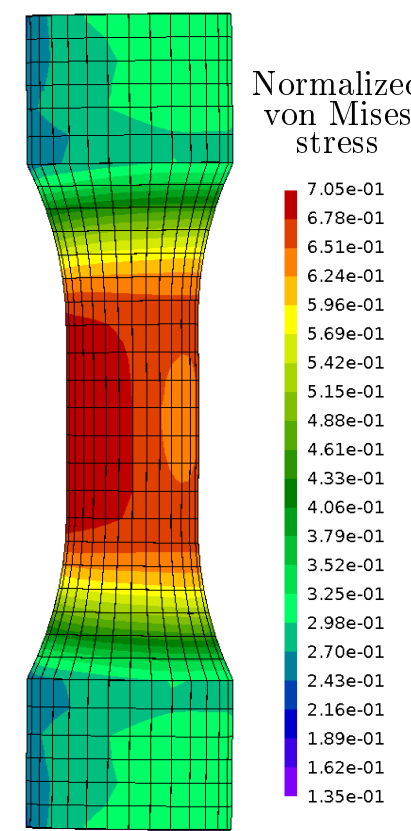

(d)

Plastic strain

$-2.03 e-03$ $-2.09 \mathrm{e}-03$
$-2.15 \mathrm{e}-03$ $-2.22 \mathrm{e}-03$ $-2.28 \mathrm{e}-03$ $-2.34 \mathrm{e}-03$ $-2.40 \mathrm{e}-03$ $-2.59 \mathrm{e}-03$ $-2.65 \mathrm{e}-03$ $-2.71 \mathrm{e}-03$ $-2.77 e-03$ $-2.83 e-03$ $-2.90 \mathrm{e}-03$ $-2.96 \mathrm{e}-03$ $-3.02 \mathrm{e}-03$ $-3.08 \mathrm{e}-03$ $-3.14 \mathrm{e}-03$

$-3.21 \mathrm{e}-03$ $-3.27 e-03$
$-3.33 e-03$ $33 \mathrm{e}-03$
Figure 9: Equivalent axial strain contours for the characterized FEA on the grade D data set as seen from (a) $+\mathrm{X}$ and (b) $-\mathrm{X}$. The normalized Von Mises equivalent stress contours as seen from both sides of the experiment are given in (c) and (d).

with $A_{0}=0.08 \mathrm{~mm}$ and $A_{1}=2$. The angle of the neutral axis changes over the simulated time according to using a normalized Young's modulus of $E=0.1 \mathrm{GPa}$ and Poisson's ratio of $\nu=0.2$. The virtual hardmetal sample further has a normalized initial yield stress of $\sigma_{0}=0.167 \mathrm{MPa}$ while the hardening model according to Equation (2) uses an initial hardening rate of $\theta_{0}=0.667 \mathrm{GPa}$, saturation stress $\sigma_{S}=0.833 \mathrm{MPa}$ and hardening exponent $n=1.5$. All of the values are normalized using the same scale factor not reported due to an ongoing memorandum of understanding regarding the proprietary nature of the alloys tested. The problem is solved using CalculiX and the following virtual data extracted from the results:

- The axial strain history of the central test section elements closest to the $0, \frac{2}{3} \pi$ and $\frac{4}{3} \pi$ radian locations along the test section circumference. This virtual data is chosen to represent the three strain gauges' histories in the original experiments.

- The total axial reaction force for all nodes on the top face of the material section. This is chosen to represent the experimental load cell data.

Since the values of the material parameters used in the virtual experiment are known, an inverse identification problem can be solved to determine the accuracy with which these known parameters and boundary conditions can be retrieved. Such an investigation gives important insight as to whether the data available is sufficient to distinguish between the effects attributed to the work hardening and the boundary conditions respectively. The elastic material parameters are again not included in the inverse problem. 
As with the real experimental data, the average axial strain is computed by simple averaging of the three axial strain histories. If a volume conserving cylinder is assumed, it allows the calculation of the true stress. As done in Figure 3 for the true material samples, the material parameter values were then estimated using this equivalent stress-strain curve. This leads to an initial yield stress $\sigma_{0}=0.140 \mathrm{MPa}(16.17 \%$ less than the true $0.167 \mathrm{MPa}$ value), initial hardening $\theta_{0}=0.618 \mathrm{GPa}$ $(7.35 \%$ less than the true value of $0.667 \mathrm{GPa})$, saturation stress $\sigma_{S}=0.795 \mathrm{MPa}(4.56 \%$ less than $0.833 \mathrm{MPa})$ and exponent $n=1.473$ (1.8\% less than 1.5). These material parameter values are used as the initial guess to the FEA based parameter estimation as in the grade $\mathrm{D}$ case of the previous section.

Two different inverse parameter characterizations are performed using the virtual experiment data:

- Fully parametrized. This test is performed to test the true invertibility of the fully parametrized numerical experiment. The parameters associated with initial yield $\left(\sigma_{0}\right)$ and plastic hardening $\left(\theta_{0}\right.$, $\sigma_{S}$ and $n$ ) are determined alongside the boundary function parameters $\left(A_{0}, A_{1}, B_{0}, B_{1}, B_{2}, C_{0}\right.$ and $C_{1}$ ) in Equations (8) to (10). Therefore we have assumed an identical parameterization to the functional forms used to generate the data, a situation that is unlikely to ever occur in reality.

- Discrete approximation. This test is performed to investigate the accuracy with which the material parameters of interest is characterized despite unknown boundary function parameterization. The parameters associated with initial yield $\left(\sigma_{0}\right)$ and plastic hardening $\left(\theta_{0}, \sigma_{S}\right.$ and $\left.n\right)$ are again determined, but now using piecewise linear approximations to the boundary value functions. The inverse characterization is started with linear approximations for $A(t)$ and $C(t)$, and a constant approximation for $B(t)$. Once the estimation stage has converged, each piecewise linear approximated line segment is divided in two. The initial conditions to the next estimation stage is set up by interpolating from a previous stage result and the process continues. As before, the iterative refinement and inverse analysis are done in six stages.

The initial conditions, objective function, final objective function values, material parameter values, boundary conditions and iterations needed for the different characterizations are given in Table 2. The objective function value and initial conditions for the fully parametrized inverse characterization is tabulated in column three $\left(X_{0}\right)$ of Table 2 with the converged values after 2376 function evaluations in column four $\left(X^{*}\right)$. From the fully parametrized result, the problem seems uniquely invertible with the material parameter and boundary function values converging to the known true values.
Investigation of the six stage refinement ( $X_{i}$ for $i \in$ $0,1, \ldots, 6)$ of the piecewise linear boundary value function approximation also produce the desired result. In the first stage $\left(X_{1}\right)$, using 7 variables (four material and three boundary values), the converged material parameter values are a worse approximation of the true parameter values as compared to the trivial postprocessing guess $\left(X_{0}\right)$. For each subsequent piecewise linear approximation refinement however the material parameters of interest and boundary function form gets closer to their true values. The true boundary functions in Equations (8) to (10), as well as the converged piecewise linear forms at the start and end of each refinement inverse analysis stage, are presented in Figures $10(\mathrm{a})$ to $(\mathrm{c})$.

According to column six of Table 2 and Figure 10, the use of a single variable per boundary function is not effective. From two piecewise linear segments onward there is sufficient flexibility to modify the shape of the functions modeled in such a way that they approach the true shape. Additional refinement and flexibility result in even better capturing of the form of the underlying boundary functions despite no prior information on shape or parametrization provided to the numerical optimization algorithm. This along with the material parameter values of interest approaching their known values indicate that the process of iterative piecewise linear refinement and inverse characterization has the desired effect. Even if the parametrization or form of the actual boundary functions are unknown, the material parameter values can be determined accurately using the proposed iterative inverse estimation process, making use of multiple piecewise linear approximations.

Over the six stage refinement, there is a visible trend in the converged objective function value and the number of function evaluations, as a function of the number of discrete values used to approximate the boundary function forms. The objective function value and the number of function evaluations in Table 2 are also plotted in Figures 11(a) and (b). The percentage error of the material parameter values from their true values, is illustrated using the black lines in Figure 11(c). The integral error between the piecewise linear approximated and true parametrized functions in Equations (8) to (10) are given as the red lines. Given more boundary modeling flexibility, the material parameters and boundary function approximation are seen to converge towards the true result. This is however at a significant computational cost, considering that the full six stage characterization process on the virtual data required 26537 FEA simulations.

Despite the high computational demands, this test on virtual data presents useful insight. The investigation confirms that the correct material parameters may still be obtained despite the non-ideal experimental data arising from an unknown compressive instability. However, this was illustrated for the somewhat idealistic 
Table 2: Convergence and solution to the fully parametrized and discrete approximation inverse problem using virtual data

\begin{tabular}{|c|c|c|c|c|c|c|c|c|c|c|}
\hline \multirow[t]{2}{*}{ Variable : Scale [Unit] } & \multirow[t]{2}{*}{ Actual } & \multicolumn{2}{|c|}{ Parametrized } & \multicolumn{7}{|c|}{ Discrete Approximation - Refinement Stage } \\
\hline & & $X_{0}$ & $X^{*}$ & $X_{0}$ & $X_{1}$ & $X_{2}$ & $X_{3}$ & $X_{4}$ & $X_{5}$ & $X_{6}$ \\
\hline Objective Value & & 30.057 & 0.0031 & 52.356 & 11.202 & 4.6917 & 1.1998 & 0.3205 & 0.0378 & 0.0104 \\
\hline Number of Variables & & 11 & 11 & 7 & 7 & 11 & 17 & 29 & 53 & 101 \\
\hline Iterations & & - & 2376 & - & 1103 & 1445 & 2088 & 4202 & 6601 & 11098 \\
\hline \multicolumn{11}{|l|}{ Material } \\
\hline$\sigma_{0}: 1 \mathrm{e} 3[\mathrm{MPa}]$ & 0.1667 & 0.1402 & 0.1667 & 0.1402 & 0.3844 & 0.1848 & 0.1676 & 0.1638 & 0.1654 & 0.1659 \\
\hline$\theta_{0}: 1 \mathrm{e} 3[\mathrm{GPa}]$ & 0.6667 & 0.6183 & 0.6666 & 0.6183 & 0.4245 & 0.6211 & 0.6263 & 0.6758 & 0.6665 & 0.6679 \\
\hline$\sigma_{S}: 1 \mathrm{e} 3[\mathrm{MPa}]$ & 0.8333 & 0.7950 & 0.8333 & 0.7950 & 0.4313 & 0.7753 & 0.8595 & 0.8295 & 0.8331 & 0.8334 \\
\hline$n$ & 1.5 & 1.4730 & 1.4999 & 1.4730 & 1.3521 & 1.1249 & 1.5295 & 1.4781 & 1.4977 & 1.5017 \\
\hline \multicolumn{11}{|l|}{ Boundary } \\
\hline$A_{0}: 1 \mathrm{e}-2$ & 8 & 10 & 8.0000 & - & - & - & - & - & - & - \\
\hline$A_{1}$ & 2 & 1.5000 & 2.0003 & - & - & - & - & - & - & - \\
\hline$A^{*}(1): 1 \mathrm{e}-2$ & 8 & - & - & 10.000 & 3.3612 & 6.6515 & 8.6012 & 7.8884 & 7.9979 & 8.0069 \\
\hline$B_{0}:$ [rads] & 4.3 & 4.0000 & 4.3028 & - & - & - & - & - & - & - \\
\hline$B_{1}:$ [rads] & 0.5 & 0.1000 & 0.5027 & - & - & - & - & - & - & - \\
\hline$B_{2}$ & 3 & 2.0000 & 3.0101 & - & - & - & - & - & - & - \\
\hline$B^{*}(0):$ rads $]$ & 4.3 & - & - & 4.0000 & 3.8320 & 4.4694 & 4.7367 & 4.5729 & 4.4799 & 4.3598 \\
\hline$B^{*}(1):$ rads] & 3.8 & - & - & 4.0000 & 3.8320 & 3.7922 & 3.7824 & 3.8008 & 3.8001 & 3.7996 \\
\hline$C_{0}: 1 \mathrm{e}-1[\mathrm{~mm}]$ & 5 & 6.0000 & 5.0001 & - & - & - & - & - & - & - \\
\hline$C_{1}$ & 1.5 & 1.0000 & 1.5000 & - & - & - & - & - & - & - \\
\hline$C^{*}(1): 1 \mathrm{e}-1[\mathrm{~mm}]$ & 3.8843 & - & - & 4.0000 & 2.2577 & 3.6319 & 3.9167 & 3.8760 & 3.8845 & 3.8849 \\
\hline
\end{tabular}

scenario where the chosen material model is a perfect match to the true stress-strain curve. In a more realistic setting where the chosen material model does not match the true stress-strain curve perfectly, the fitting error will be spread between the strain curves and the force curve. Consequently, the boundary function approximations as well as the material parameter values will both contain errors as compared to their true values. If continued refinement of the boundary does not eventually result in an acceptably small fitting error, this provides an indication that the assumed material model is inadequate.

\section{Results on Actual Data}

The simultaneous estimation of material parameter values and boundary function approximations, using the proposed multiple stage estimation process, is now applied to all the experimental data. The results in the previous section instills confidence that the process may be used to characterize the material model with greater accuracy than the simple post-processed characterization in Figure 3.

The numerically simulated force and strain histories for each experiment are compared to the actual data in Figure 12. The mean absolute fractional error for the force curves is $1.783 \mathrm{e}-3$, with a maximum error of $1.179 \%$ for the Grade $\mathrm{C}$ material at 97.13 seconds. The strain curves are fitted with similar accuracy, with a mean absolute fractional error of 2.312e-3. A maximum error of $2.012 \%$ is observed for the Grade B material at 102.82 seconds.

The material parameter values, estimated using the simple post-processed data and the detailed FEA based inverse identification procedure, are compared in Table 3 . The closest agreement in the estimated parameters is the saturation stress for the Grade D material, that is within $3.0 \%$ of each other. The parameter that differs the most is the initial hardening rate of the Grade A material, that differs by $48.34 \%$. On average, the simple post-processed and detailed FEA based parameter value estimates differ by $12.09 \%$.

Even though the material parameter values differ on average by $12.09 \%$, the true indicator of similarity is the stress-strain curves produced when using these parameter values. Figure 13 depicts the resulting stressstrain curves. For each material parameter set found by simple post-processing and detailed FEA identification, the stress is plotted as a function of plastic strain up to $2 \%$. The average differences between the curves for Grade A to D are 13.94\%, 6.84\%, 5.27\% and $4.06 \%$ respectively. The gradual increase in accuracy is no coincidence, since the materials are ordered from hardest to softest. Note that the Grade A experiment induces less than $0.5 \%$ plastic strain on average, while the Grade D experiment induces about $1.7 \%$ plastic strain (see Figure 3(b)). Therefore more data is available deeper into the plastic strain regime to calibrate the material parameters for Grades C and D, as compared to Grades A and B. Nevertheless, the differences observed in the material parameter values in Table 3 , as well as the differences observed in the stress-strain curves in Figure 13, provides a compelling argument in favor of detailed FEA based material parameter esti- 


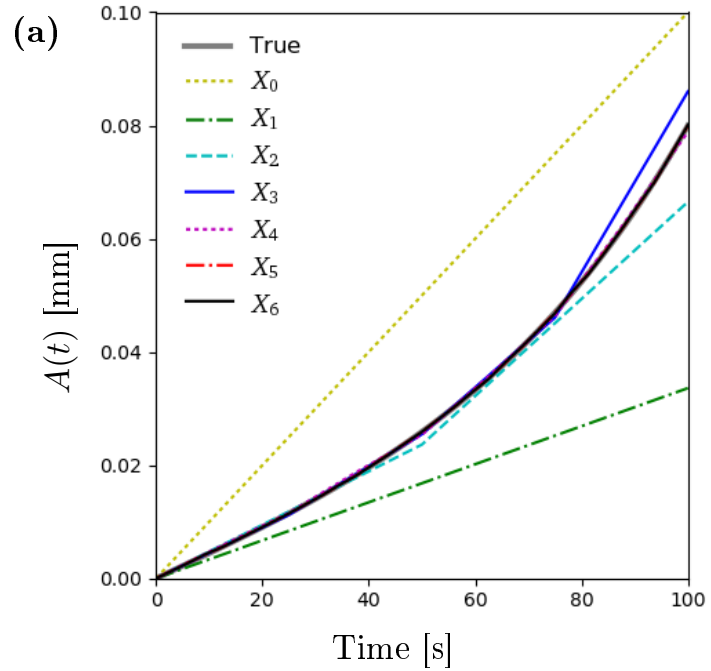

(b)
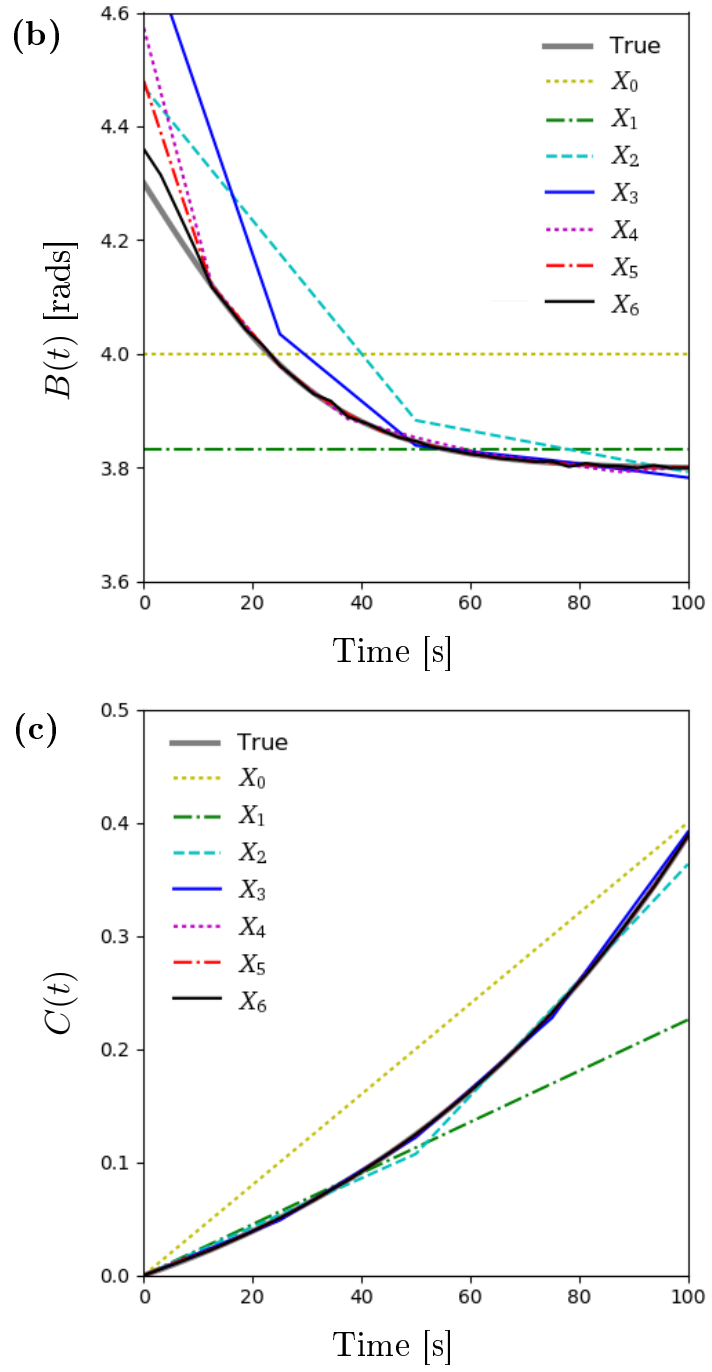

Figure 10: Boundary functions associated with (a) Equation (8), (b) Equation (9) and (c) Equations (10). The initial conditions using a single value unknown per function is shown using a dashed yellow line. The converged piecewise linear approximation to each function is then shown as a result of six incremental refinement and inverse analysis stages. NOTE: The objective function is only evaluated for data points after 10 seconds to give the elastic data less prevalence. This could explain the inability to capture $B(t)$ for $t \leq 10$. (a)

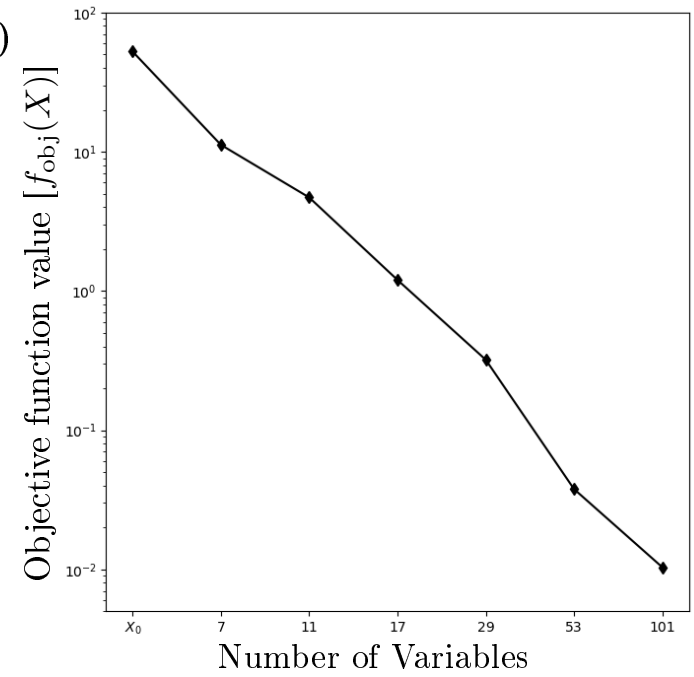

(b)

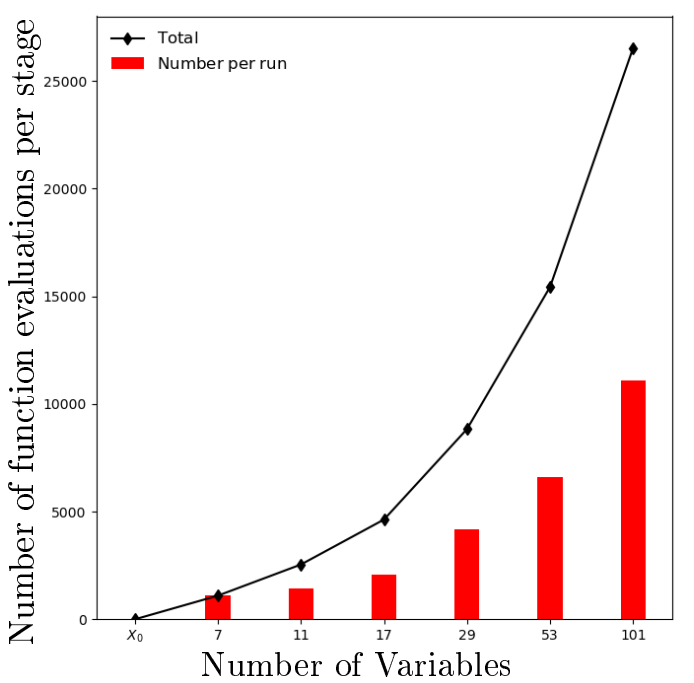

(c)

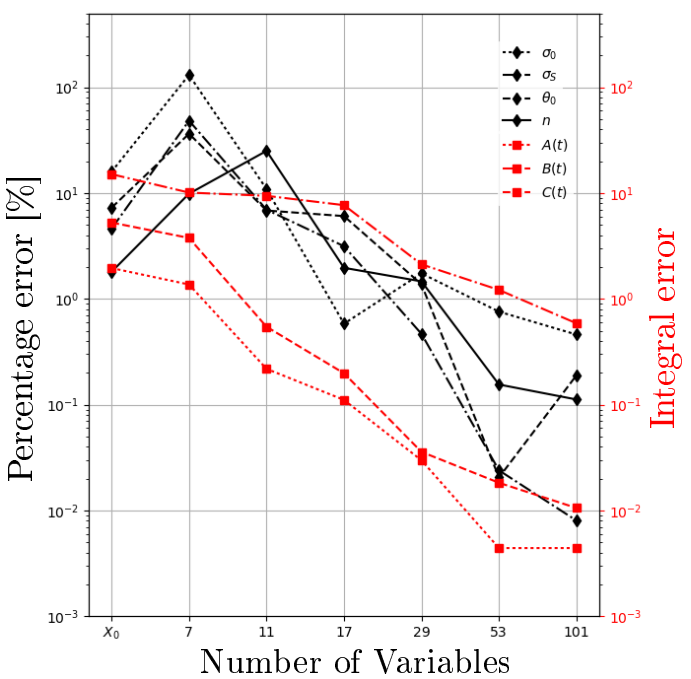

Figure 11: Convergence and function evaluations required during the six stage refinement and characterization on virtual experiment data. (a) Objective function value for the initial and each converged variable values. (b) Number of function evaluations required per stage. (c) Material parameter error and integral boundary function error at the start and end of each stage. 


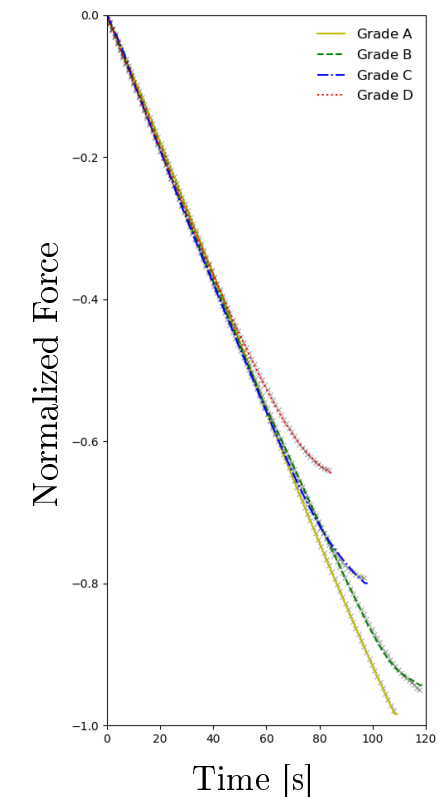

(a)

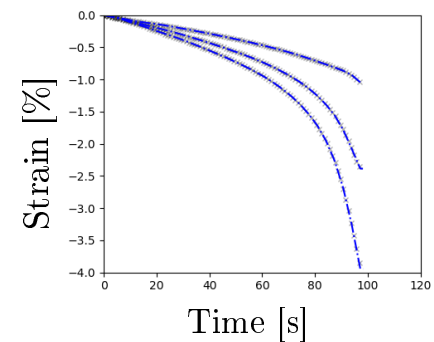

(d)

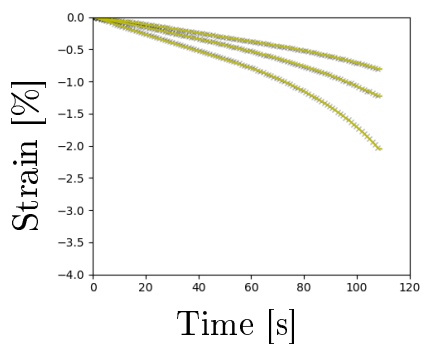

(b)

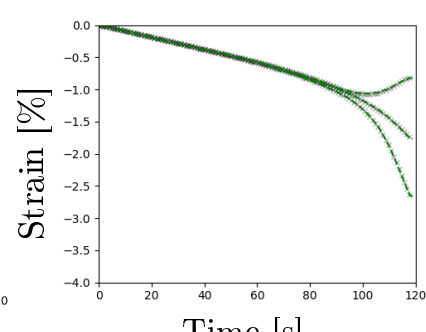

(c)

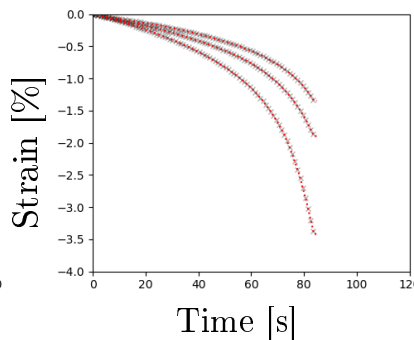

(e)

Figure 12: (a) Fit between the normalized experimental load cell data $(+)$ and total axial reaction force simulated (lines). The modeled and experimental strain histories are also compared for all three strain gauges of the hardmetal grade (b) A, (c) B, (d) C and (e) D non-ideal compression experiment.

Table 3: Material model parameters as a result of simple post-processing and detailed FEA inverse identification

\begin{tabular}{|c|c|c|c|c|c|}
\hline & $\begin{array}{l}\text { Average } \\
\text { (Normalized) }\end{array}$ & $\begin{array}{l}\mathrm{A} \\
(\text { Scale }\end{array}$ & $\begin{array}{l}\text { B } \\
\text { param }\end{array}$ & $\begin{array}{l}\mathrm{C} \\
\text { ter val }\end{array}$ & $\begin{array}{l}\mathrm{D} \\
e s)\end{array}$ \\
\hline \multicolumn{6}{|c|}{ Simple post-processing } \\
\hline$\sigma_{0}$ & $0.374 \mathrm{MPa}$ & 1.523 & 1.249 & 0.623 & 0.605 \\
\hline$\theta_{0}$ & $0.787 \mathrm{GPa}$ & 1.298 & 1.200 & 0.871 & 0.631 \\
\hline$\sigma_{S}$ & $0.869 \mathrm{MPa}$ & 1.227 & 1.125 & 0.910 & 0.738 \\
\hline$n$ & 1.625 & 1.072 & 1.008 & 0.957 & 0.962 \\
\hline
\end{tabular}

FEA based inverse analysis

$\begin{array}{llllll}\sigma_{0} & 0.469 \mathrm{MPa} & 1.630 & 1.156 & 0.635 & 0.579 \\ \theta_{0} & 0.562 \mathrm{GPa} & 1.111 & 1.039 & 1.057 & 0.793 \\ \sigma_{S} & 1.034 \mathrm{MPa} & 1.542 & 1.041 & 0.785 & 0.632 \\ n & 1.659 & 1.529 & 0.847 & 0.790 & 0.834\end{array}$

Differences [\%] (between original, not scaled)

\begin{tabular}{lllll}
$\sigma_{0}$ & 29.29 & 14.94 & 24.49 & 18.26 \\
$\theta_{0}$ & 48.34 & 47.26 & 14.38 & 10.90 \\
$\sigma_{S}$ & 40.77 & 10.73 & 3.727 & 2.998 \\
$n$ & 37.14 & 15.30 & 17.06 & 12.19 \\
\hline
\end{tabular}

mation.

\section{Conclusions}

In this paper, the concept of simultaneous estimation of material parameter values and time varying boundary values was investigated using finite element analyses within the optimization loop. From the investigation the proposed procedure seems satisfactory in being able to more accurately determine the material parameter values than a simple post-processing procedure. The piecewise linear boundary function approximation and refinement illustrated that improved accuracy could be achieved, even when the correct boundary function parameterization is unknown. The proposed procedure does however come at an increased computational cost.

A study on a virtual experiment illustrated that using too simple an approximation on the boundary condition could result in an inadequate fit and material parameters that are far from the desired accuracy. Here it seems that the material parameters are exploited to compensate for the inadequate capture of the boundary condition. As the boundary form is given more flexibility in later stages of the refinement and refit by inverse analysis, the material parameters of interest converge to the known true values.

The effect of potential measurement errors in the data also have to be considered when characterizing material 


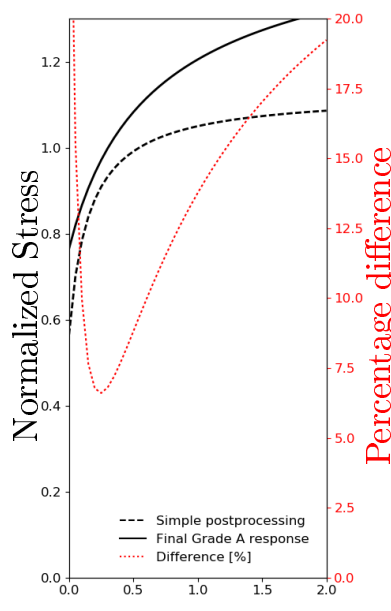

Plastic Strain $[\%]$

(a)

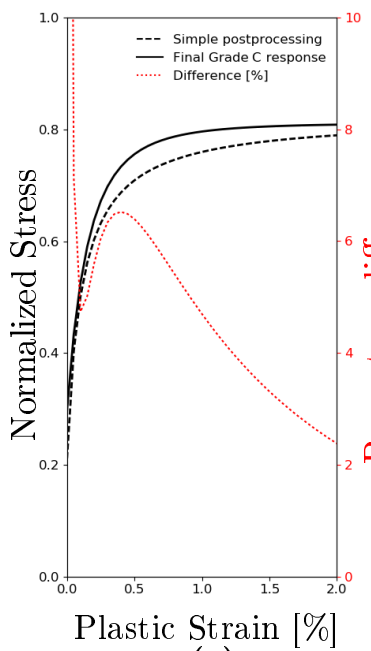

(c)

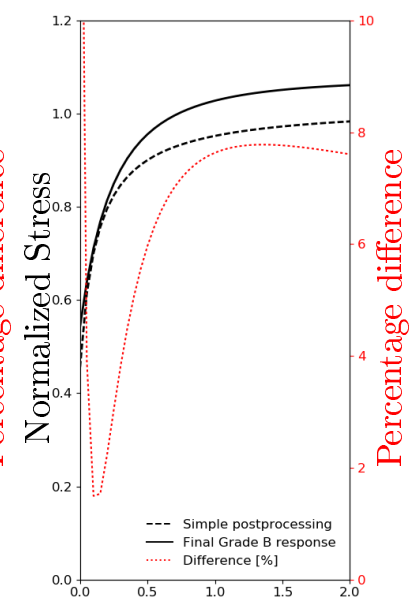

Plastic Strain [\%]

(b)

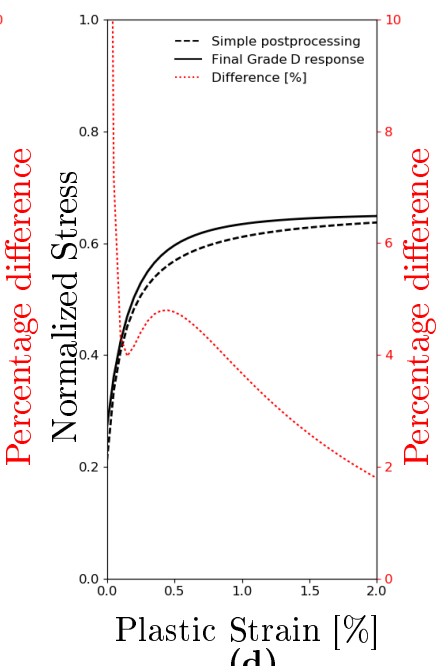

(d)
Figure 13: Comparison between hardening curves as a result of simple post-processing and detailed FEA inverse characterization for each of the different hardmetal grade samples tested. The stress vs. plastic strain curves using the two different methods are compared, including the percentage difference (red lines). (a) Grade A, (b) Grade B, (c) Grade C and (d) Grade D curves are shown.

model parameters. In earlier work, Jansen van Rensburg et al. (2014) showed the effect of a $2 \%$ Gaussian noise on the data using a similar virtual experiment to the one presented in Section 5. The material parameter sensitivity to noise is expected to be within the same order of magnitude when considering the detailed FEA and simple post-processing inverse material parameter identification.

Given similar non-ideal compression experiment data to that used in this paper, the same process as outlined here should result in material model characterization with increased accuracy as compared to a simple postprocessing method.

\section{References}

Ageno M, Bolzon G, Maier G (2009) An inverse analysis procedure for the material parameter identification of elastic-plastic free-standing foils. Structural and Multidisciplinary Optimization 38:229-243

Åkerström P, Wikman B, Oldenburg M (2005) Material parameter estimation for boron steel from simultaneous cooling and compression experiments. Modelling and Simulation in Materials Science and Engineering 13:1291-1308

ASTM C1424-04 (2004) Standard Test Method for Monotonic Compressive Strength of Advanced Ceramics at Ambient Temperature. Standard, ASTM, Philadelphia, PA

ASTM E209-00 (2010) Standard Practice for Compression Tests of Metallic Materials at Elevated Temperatures with Conventional or Rapid Heating Rates and Strain Rates. Standard, ASTM, Philadelphia, PA

ASTM E9-09 (1989) Standard Test Methods of Compression Testing of Metallic Materials at Room Temperature. Standard, ASTM, Philadelphia, PA

AZO Materials (2002) Properties: Tungsten Carbide An Overview. URL http: //www. azom.com/properties.aspx? ArticleID $=1203$

Bruhns OT, Anding DK (1999) On the simultaneous estimation of model parameters used in constitutive laws for inelastic material behaviour. International Journal of Plasticity 15:13111340

Chen X, Ashcroft IA, Wildman RD, Tuck CJ (2017) A combined inverse finite element - elastoplastic modelling method to simulate the size-effect in nanoindentation and characterise materials from the nano to micro-scale. International Journal of Solids and Structures 104-105:25-34

Dhondt G, Wittig K (1998) CalculiX:A Free Software ThreeDimensional Structural Finite Element Program. URL http: //www.dhondt.de/

Dunlay WA, Tracy C, Perrone P (1989) A Proposed Uniaxial Compression Test for High Strength Ceramics. Tech. rep., U.S. Army Materials Technology Laboratory

Gamonpilas C, Busso EP (2007) Characterization of Elastoplastic Properties Based on Inverse Analysis and Finite Element Modeling of Two Separate Indenters. Journal of Engineering Materials and Technology 129:603-608

Garbowski T, Maier G, Novati G (2012) On calibration of orthotropic elastic-plastic constitutive models for paper foils by biaxial tests and inverse analyses. Structural and Multidisciplinary Optimization 46:111-128

Ghouati O, Gelin JC (1998) Identification of material parameters directly from metal forming processes. Journal of Materials Processing Technology 80-81:560-564

ISO 4506 (1979) Hardmetals - Compression test. Standard, International Organization for Standardization, Geneva, $\mathrm{CH}$

Jansen van Rensburg GJ, Kok S, Wilke DN (2012) Simultaneous estimation of experimental and material parameters. In: EngOpt 2012 - 3rd International Conference on Engineering Optimization, Rio de Janeiro, Brazil, 1-5 July 2012

Jansen van Rensburg GJ, Kok S, Wilke DN (2014) Simultaneous boundary value and material parameter estimation using imperfect compression data. In: EngOpt 2014 - 4th International Conference on Engineering Optimization, Lisbon, Portugal, 811 September 2104

Jansen van Rensburg GJ (2016) Development and implementation of state variable based user materials in computational plasticity. Ph.D. thesis, The University of Pretoria, Pretoria, South Africa 
Jansen van Rensburg GJ, Kok S, Wilke DN (2017) Steel Alloy Hot Roll Simulations and Through-Thickness Variation Using Dislocation Density-Based Modeling. Metallurgical and Materials Transactions B, 48(5):2631-2648

Jekel CF, Venter G, Venter MP (2016) Obtaining a hyperelastic non-linear orthotropic material model via inverse bubble inflation analysis. Structural and Multidisciplinary Optimization $54: 927-935$

Jones E, Oliphant E, Peterson P (2001) SciPy: Open Source Scientific Tools for Python. URL http://www.scipy.org/

Kocks U, Tomé C, Wenk H (1998) Texture and Anisotropy. Cambridge University Press

Mahnken R, Stein E (1996) A unified approach for parameter identification of inelastic material models in the frame of the finite element method. Computer methods in applied mechanics and engineering 136:225-258

Mourad H, Bronkhorst C, Addessio F, Cady C, Brown D, Chen S, Gray G (2013) Incrementally objective implicit integration of hypoelastic-viscoplastic constitutive equations based on the mechanical threshold strength model. Computational Mechanics 53:941-955

Nelder J, Mead R (1965) A simplex method for function minimization. The Computer Journal 7:308-313

Schmaltz S, Willner K (2014) Comparison of Different Biaxial Tests for the Inverse Identification of Sheet Steel Material Parameters. Strain 50:389-403

Wang X, Li H, Chandrashekhara K, Rummel, SA, Lekakh S, Van Aken DC, O'Malley RJ (2017) Inverse finite element modeling of the barreling effect on experimental stress-strain curve for high temperature steel compression test. Journal of Materials Processing Technology 243:465-473

Wikman B, Bergman G, Oldenburg M, Häggblad HA (2006) Estimation of constitutive parameters for powder pressing by inverse modelling. Structural and Multidisciplinary Optimization 31:400-409 